\title{
Design of the uniaxial shaker with variable stiffness
}

\author{
Ondrej Chlebo ${ }^{1, *}$, Martin Sivý ${ }^{1}$, Miloš Musil ${ }^{1}$ and Michal Čekan ${ }^{1}$ \\ ${ }^{1}$ Slovak University of Technology in Bratislava, Nam. Slobody 17, 81231 Bratislava, Slovakia
}

\begin{abstract}
During the vibration testing, various types of vibration drivers of different structures are used. Electrodynamic drivers are generally used for smaller excitation forces whereas for bigger excitation forces hydraulic drivers are used. The main disadvantage of drivers can be found in their size. One of the possibilities for size reduction may be a suitable engineering design. The paper deals with the design of a small uniaxial driver which uses an inertial driver as a source of the excitation force. The structure considers the requirements for stiffness changes of the whole system with the aim of available frequency range tuning.
\end{abstract}

\section{Introduction}

Every kinematic excitation system is composed of three basic elements. The first is the amplifier which is responsible for transferring sufficient energy into the working element, which is coincidentally the second part of the system. The third element is the fixing structure which is responsible for fixing the studied part and connecting it to the working element which must allow for motion in at least one dimension. The paper deals with a uniaxial kinematic driver. This driver is a system with one degree of freedom which is excited by the harmonic force $F_{0} \sin \omega t$. This system is possible to represent by a simple motion equation in the form [1-4]:

$$
m \ddot{x}+b \dot{x}+k x=F_{0} \sin \omega t
$$

where $m$ is the mass of the system, $b$ is the damping matrix, and $k$ is the stiffness matrix. Equation (1) can be used to describe the natural frequency of a damped system, provided that the damping is relatively low, by:

$$
\omega_{d}=\sqrt{\frac{k}{m}-\left(0.5 \frac{b}{m}\right)^{2}}
$$

From this equation (2) it can be seen that the natural frequancy is dependent on three parameters. Other than the stiffness and damping properties of the system, mass has a significant effect on the frequency of the system. However unlike the other two parameters, the mass of the system is affected by the mass of the studied object as well. Therefore, it is desirable to account for system tuning within the structure of the driver.

\footnotetext{
*Corresponding author: ondrej.chlebo@stuba.sk
} 


\section{The structural design of the driver}

The system TIRAvib TV 51165-IN was used as a source of kinematic excitation. This system is composed of a high-performance amplifier and inertial electrodynamic air cooled driver. This driver was fixed horizontally to the right side of the mounting table. Although the table must be fixed, it should allow for motion in a particular direction. One possibility is to use a pre-stressed linear bearing, this however does not allow for tuning of the system stiffness. Another possibility is to mount the vibrating table on springs such as leaf springs as seen in Fig. 1. Doing this allows for stiffness tuning by changing the length of the leaf spring such that the natural frequency is shifted away from the desired working frequencies of the system [5].

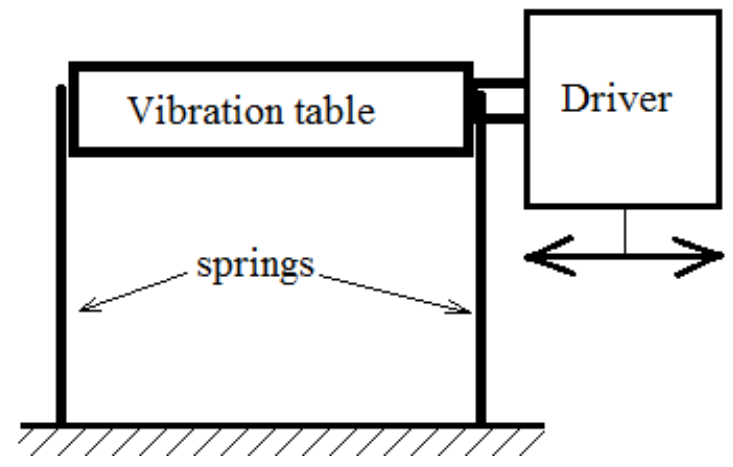

Fig. 1. Schematic model of the structural driver.

The driver is composed of mainly the vibrating table which is a welded steel ribbed plate with mounting grooves. Leaf springs are bolted to the table and fixed to a steel base. The leaf springs allow for significant movement in one direction which will correspond to the first resonant frequency of the vibrating system. Changing the length of the leaf springs changes the stiffness of the excited system.

\section{FEA of the system}

The first step was to design and analyse the vibrating plate of the driver. T80 profile was welded at its end to steel sheet. Through finite element analysis the structure was favourably reinforced to push the natural frequencies away from the measurement range. The final configuration of the table, as well as its corresponding natural frequencies, can be seen in Fig. 2. The first natural frequency of the vibrating table was $446 \mathrm{~Hz}$. This frequency is varying depending on the number and placement of test piece mounting brackets as well as the character of the test piece itself. 

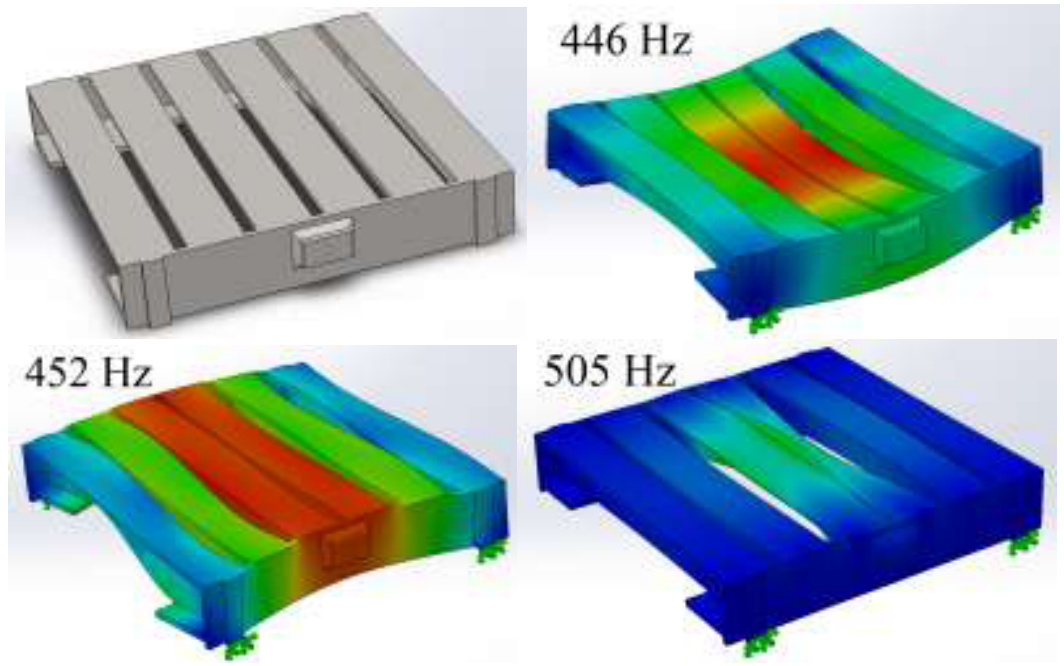

Fig. 2. Model of the vibration table and the first three modes.

The table was then mounted onto leaf springs. The natural frequency, and usable range, of the system is dependent on the length of these springs because they represent the least stiff member of the system. For this reason, analysis was performed with the table at the maximum and mid height of the springs. The results can be compared in Fig. 3. In this figure the effects of the leaf springs on the natural frequency of the system is clear, where the table acts as a rigid member at the first few natural frequencies of the system. This was done purposefully to satisfy the measurement range up to $100 \mathrm{~Hz}$.
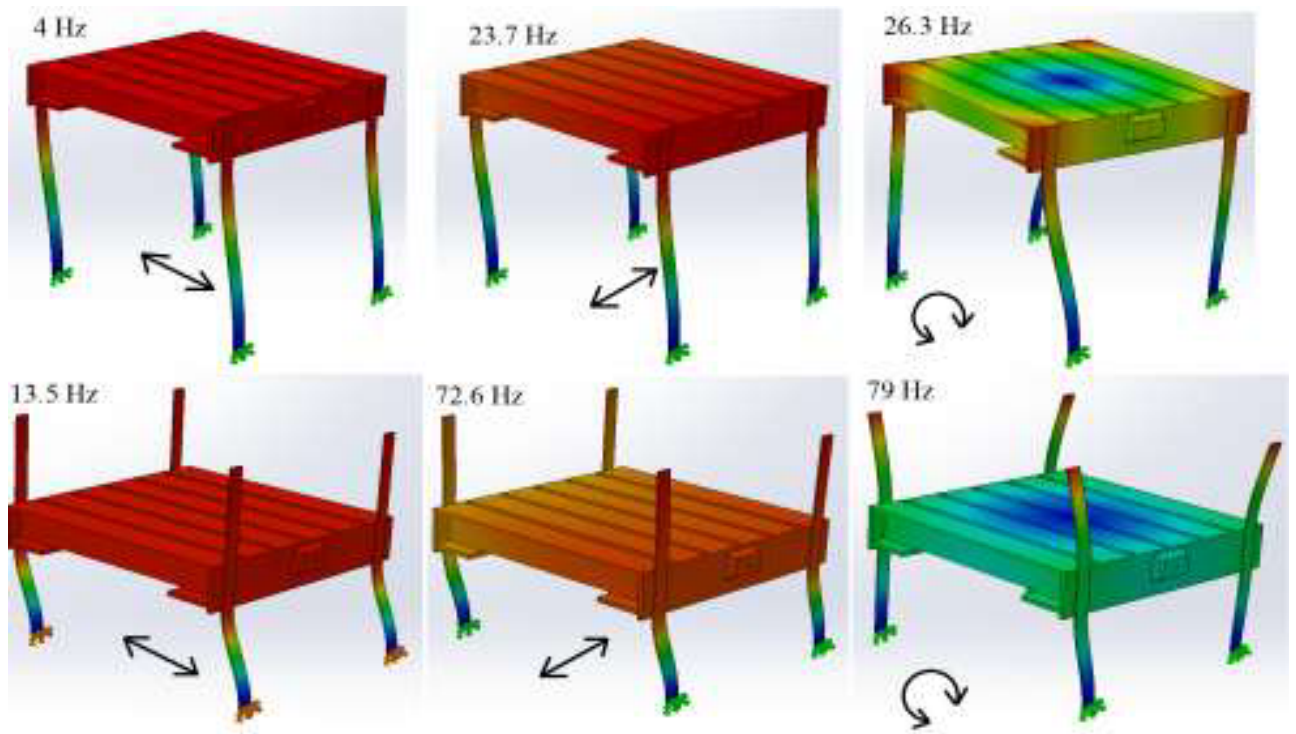

Fig. 3. Mode shapes of the vibrating system for the first three natural frequencies, top row of low stiffness and medium stiffness is the bottom row. 


\section{Experimental verification of the natural frequencies}

After analysis, the vibrating table was built and tested. Modal analysis was performed to verify the numerical results (detail of the analysis is given in [6]). The modal analysis was performed using the measuring card B\&K PULSE 3053-B-120 with accelerometer B\&K 4534-B-001. The source was generated using an impact hammer PCB 086c03. Measured data was processed in the PULSE 19 software. Results can be seen in Fig. 4.
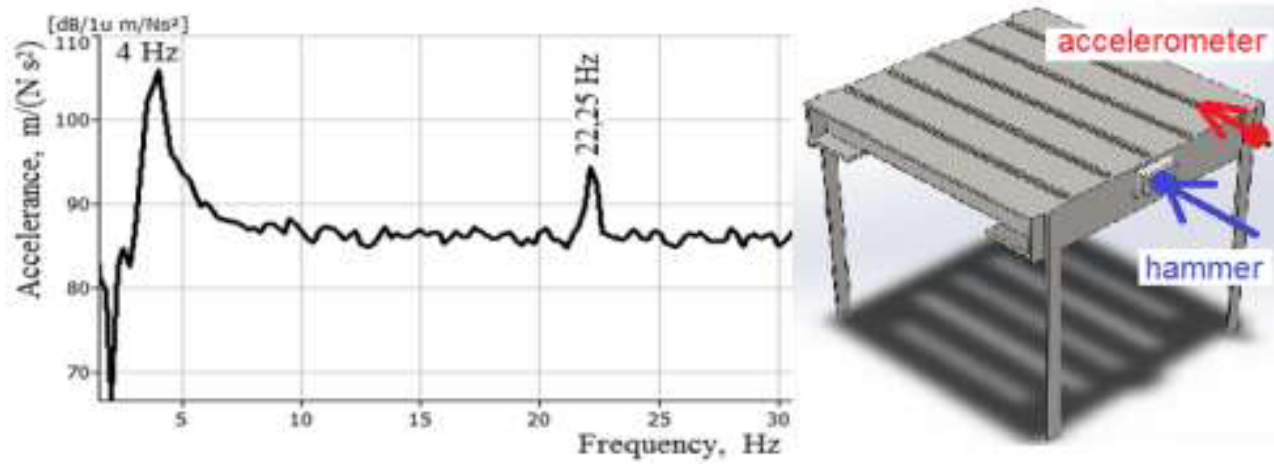

Fig. 4. Natural frequencies of the vibration system, first and third natural frequencies (the second natural frequency cannot be measured in this direction).

\section{Conclusions}

The purpose of this contribution was to design a vibrating system which could be utilized as a source of inertial excitation. During the design, the main requirement was to account for changing stiffness in order to accommodate the desired range of measurement. Based on numerical analysis, the table was optimized and built. Natural frequencies were confirmed using experimental modal analysis. In the low stiffness configuration, the first natural frequency was found at $4 \mathrm{~Hz}$ which corresponds to the numerical analysis. The third natural frequency was measured at $22.25 \mathrm{~Hz}$ whereas the numerical analysis computed the natural frequency at $23.7 \mathrm{~Hz}$. This slight discrepancy, amongst other things, may be due to small variations in the mounting to the leaf springs.

This work was supported by the STU Grant scheme for Support of Young Researchers entitled Design and Optimization of the Measuring Equipment for Vibration Tests.

\section{References}

1. S. Braun, D. Ewins, S. S. Rao, Encyclopedia of Vibration (Academic Press, New York, 2002)

2. R. Brepta, L. Pust, F. Turek, Mechanické kmitání (Sobotáles, Praha, 1994)

3. M. Musil, Základy dynamiky strojov s Matlabom (STU, Bratislava, 2013)

4. M. Musil, M. Zuščík, Aktívne a semiaktívne odpruženie vozidla (STU, Bratislava,2012)

5. M. Nad', Modification of Modal Characteristics of Vibrating Structural Elements, (Scientific Monographs, Köthen,2010)

6. S. Žiaran, V. Kompiš, V. Mišun, J. Zajac, Technická diagnostika (STU, Bratislava, 2013) 